\title{
A Finite-Difference Simulation Method for 2D Flows about Bodies of Arbitrary Configuration
}

\author{
by Young-Gill Lee*, Member \\ Hideaki Miyata*, Member
}

\begin{abstract}
Summary
A finite-difference method is developed for a viscous flow about a body of arbitrary configuration. The no-slip body boundary conditions are approximately implemented in the framework of a rectangular grid system. Three examples of simulation, that is, a flow about a car, a flow past two bridges and a flow about a cylinder in waves, demonstrate that this method is useful for the simulation of a viscous flow about a body of complex geometry to which a boundary-fitted coordinate system finds difficulties in the generation.
\end{abstract}

\section{Introduction}

In numerical simulations, one of the major difficulties arises from the grid systems. The boundary-fitted coordinate system is currently used and its effectiveness is widely recognized. However, the process of grid generation about a body of complex geometry requires an elaborate labor and significant amount of time. An inadequate grid system straightforwardly leads to the break.down of the solution.

The MAC method ${ }^{1)}$ was the first to solve fluid flows with complex geometry in the framework of the rectangular grid system. Viecelli ${ }^{2}$ improved the MAC method by implementing the free-slip body boundary conditions on a $2 \mathrm{D}$ body of arbitrary geometry. Miyata and Nishimura ${ }^{3 / 4)}$ extended this idea to the fully $3 \mathrm{D}$ problem of a flow about an advancing ship involving free-surface waves. Both $2 \mathrm{D}$ and $3 \mathrm{D}$ wave breaking simulations are performed by Miyata and his co-workers in the framework of inflexible rectangular grid systems ${ }^{5 / 11}$. These methods have been applied to a variety of wave problems mostly in the fields of naval architecture and ocean engineering. Although the no-slip body boundary conditions are approximately implemented in some of the above works ${ }^{(0) 11)}$, it sometimes gives excessive amount of error and as a result the viscous flow that emanates from the body boundary is contaminated.

The objective of the present paper is to devolop a new numerical method which can simulate a viscous flow about a body of complex geometry in the framework of a finite-difference method based on the Navier-Stokes equations in the rectangular staggered grid system.

* Department of Naval Architecture and Ocean Engineering, The University of Tokyo, Tokyo, Japan
The no-slip body-boundary conditions are carefully implemented considering the divergence of a boundary cell. The flow is restricted within the $2 \mathrm{D}$ cases, but the presence of the free-surface, which may give wave breaking motions, is considered.

\section{Computational procedure}

\subsection{Algorithm}

The computational procedure is similar to the previous work (TUMMAC-Vbk ${ }^{10)}$ ) except for the introduction of the higher-order differencing scheme, the subgrid-scale turbulence model and the grid with variable spacing in the vertical direction. The governing equations are the Navier-Stokes equations and the continuity equation in the case of a $2 \mathrm{D}$ incompressible fluid, and represented in the Cartesian coordinates as follows.

$$
\begin{aligned}
& \frac{\partial u}{\partial t}+u \frac{\partial u}{\partial x}+w-\frac{\partial u}{\partial z}=-\frac{\partial \phi}{\partial x}+\nu\left[\frac{\partial^{2} u}{\partial x^{2}}+\frac{\partial^{2} u}{\partial z^{2}}\right], \\
& \frac{\partial w}{\partial t}+u \frac{\partial w}{\partial x}+w \frac{\partial w}{\partial z}=-\frac{\partial \phi}{\partial z}+\nu\left[\frac{\partial^{2} w}{\partial x^{2}}+\frac{\partial^{2} w}{\partial z^{2}}\right]+g \\
& \frac{\partial u}{\partial x}+\frac{\partial w}{\partial z}=0,
\end{aligned}
$$

where $\phi=P / \rho, P$ is the pressure and $\rho$ is the density, $u$ and $w$ are the velocity components in the $x$ and $z$ directions, respectively, and $\nu$ is the kinematic viscosity while $g$ is the gravitational acceleration which is ignored in the cases without the free-surface.

The computational domain is discretized into a staggered rectangular inflexible mesh system, with constant spacing in the horizontal direction while variable in the vertical so as to have finer mesh near the body and the free-surface.

The principal differencing schemes used in this finitedifference method are the Adams-Bashforth diff- 
erencing in time and the third-order upstream or second-order centered differencing in space.

\subsection{Difference Scheme}

The Poisson equation for the pressure is solved as a boundary value problem by the Successive Over Relaxation (SOR) method except in the boundary cells. The computation of the pressure in the boundary cells is described in detail in the subsequent section.

For the difference approximation of the convective terms the fourth-order accurate centered scheme combined with the fourth-derivative artificial dissipation term is employed. In equally spaced grid system one of the advective terms is expressed as

$$
\begin{aligned}
& \left\langle u \frac{\partial u}{\partial x}\right\rangle_{i+\frac{1}{2}, k} \\
& =u_{i+\frac{1}{2}, k} \frac{-u_{i+\frac{5}{2}, k}+8\left(u_{i+\frac{3}{2}, k}-u_{i-\frac{1}{2}, k}\right)+u_{i-\frac{3}{2}, k}}{12 \Delta x} \\
& \quad+\left|u_{i+\frac{1}{2}, k}\right| \alpha_{4} \frac{u_{i+\frac{5}{2}, k}-4 u_{i+\frac{3}{2}, k}+6 u_{i+\frac{1}{2}, k}-4 u_{i-\frac{1}{2}, k}+u_{i-\frac{3}{2}, k}}{24 \Delta x}
\end{aligned}
$$

The magnitude of the artificial dissipation is controlled by the parameter $\alpha_{4}$.

In the variable mesh system the above differencing scheme becomes a little more complicated to maintain the third-order accuracy. The first derivative of the advective term is generally written as

$$
\left\langle\frac{\partial u}{\partial z}\right\rangle_{k}=a_{2} u_{k+2}+a_{1} u_{k+1}+a_{0} u_{k}+a_{-1} u_{k-1}+a_{-2} u_{k-2} .
$$

Here, the subscript for the horizontal location is dropped for simplicity. Eq. ( 5 ) becomes a third-order differencing scheme, which is equivalent to the fourth-order centered differencing combined with the fourthderivative dissipation term, provided the coefficients satisfies the following equation.

$$
\begin{aligned}
& {\left[\begin{array}{cccc}
1 & 1 & 1 & 1 \\
\left(\Delta z_{k}+\Delta z_{k+1}\right) & \Delta z_{k}-\Delta z_{k-1} & -\left(\Delta z_{k-1}+\Delta z_{k-2}\right) \\
\left(\Delta z_{k}+\Delta z_{k+1}\right)^{2} \Delta z_{k}{ }^{2} & \Delta z_{k-1}{ }^{2} & \left(\Delta z_{k-1}+\Delta z_{k-2}\right)^{2} \\
\left(\Delta z_{k}+\Delta z_{k+1}\right)^{3} \Delta z_{k}{ }^{3}-\Delta z_{k-1}{ }^{3}-\left(\Delta z_{k-1}+\Delta z_{k-2}\right)^{3}
\end{array}\right]\left[\begin{array}{l}
a_{2} \\
a_{1} \\
a_{-1} \\
a_{-2}
\end{array}\right]} \\
& =\left[\begin{array}{c}
-a_{0} \\
1 \\
0 \\
0
\end{array}\right]
\end{aligned}
$$

When $a_{0}$ is set at $\alpha_{4} / 4 \cdot \Delta z_{k}$, this differencing scheme corresponds to the one expressed by Eq. (4). In this study $\alpha_{4}$ is set at 6.0 , as a result of compromising between stability and accuracy properties. In the vicinity of the boundaries, where sufficient number of velocity points are not available, the combination of the second-order centered and the donor-cell schemes or the first-order upstream scheme is used depending on the number of available velocity points.

\section{3 Turbulence Model}

The subgrid-scale (SGS) turbulence model ${ }^{12)}$ is incorporated into the present method so that a high Reynolds number flow involving vortices may be well simulated. In this study the SGS model is modified into a two- dimensional form and used in a very straightforward manner. Although the grid spacing is not sufficiently small, the simulated results in the succeeding section demonstrate the appropriateness of its use in the resolution of relatively large-scale vortical motions.

The SGS eddy viscosity coefficient $\nu_{s}$ is given in the present $2 \mathrm{D}$ case as

$$
\left(\nu_{s}\right)_{i, k}=\left(C_{1} \Delta_{i, k}\right)^{2}\left[\left(\frac{\partial u}{\partial z}\right)^{2}+\left(\frac{\partial w}{\partial x}\right)^{2}+2 \frac{\partial u}{\partial z} \frac{\partial w}{\partial x}\right]_{i, k}^{1 / 2}
$$

where

$$
\Delta_{i, k}=\left(\Delta x \cdot \Delta z_{k}\right)^{1 / 2}
$$

Then, the turbulent kinetic energy $K$, the turbulent pressure $P S$ and the turbulent shear stress $\tau_{s}$ are respectively given by the following equations.

$$
\begin{aligned}
& K_{i, k}=\left(\nu_{s}\right)_{i, k}^{2} /\left(C_{0} \Delta_{i, k}\right)^{2} \\
& \left(P_{s}\right)_{i, k}=2 / 3 K_{i, k} \\
& \left(\tau_{s}\right)_{i, k}=\left[\nu_{s}\left(\frac{\partial u}{\partial z}+\frac{\partial w}{\partial x}\right)\right]_{i, k}
\end{aligned}
$$

Consequently, the following momentum terms due to turbulence are incorporated into the Navier-Stokes equations.

$$
\begin{array}{ll}
\frac{\partial}{\partial x}\left(-P_{s}+\tau_{s}\right)_{i, k} & \text { in } x \text {-direction, } \\
\frac{\partial}{\partial z}\left(-P_{s}+\tau_{s}\right)_{i, k} & \text { in } z \text {-direction. }
\end{array}
$$

The constants $C_{0}$ and $C_{1}$ are set at 0.094 and 0.10 , respectively.

\section{4 Body Boundary Condition}

The configuration of a body is represented by a succession of segments and the cells are flagged and classified into full-of-fluid cells ( $\mathrm{F}$-cells), body boundary cells ( $\mathrm{B}$-cells) and empty cells (E-cells). A body boundary cell is a cell that contains a segment of the body boundary and the empty cell is a cell that does not contain fluid portion. As the boundary cells must be continuously located along the body boundary, one of the neighbouring F-cells is newly defined as a B-cell as seen in Fig. 1.

In the previous works ${ }^{8) 10)}$ the velocities inside the

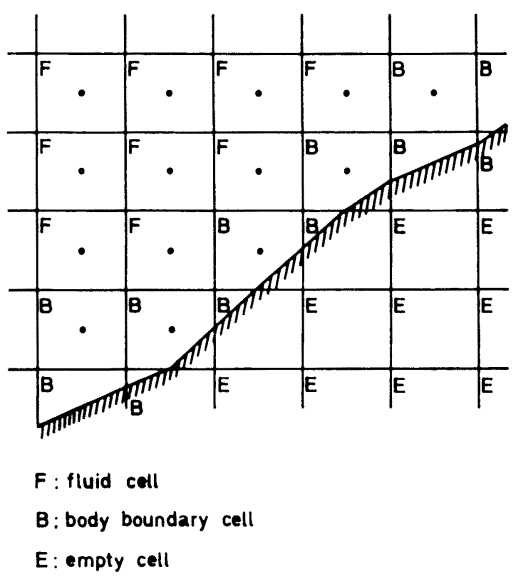

Fig. 1 Cell flagging. 


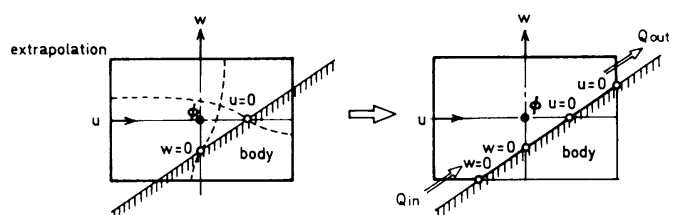

Fig. 2 Schematic sketch for no-slip body boundary conditions.

boundary are extrapolated keeping the magnitude of the velocities on the segment to be zero and then the pressure of the boundary cell is calculated by the zerodivergence condition with these velocities as shown in Fig. 2 (left). However, the extrapolated velocities are physically meaningless and this often leads to the occurrence of unrealistic pressure or the break-down of the solution. Therefore, in this study velocities inside the body are not extrapolated, but instead, the zero-velocity on the boundary is directly made use of for the implementation of the zero-divergence condition of the boundary cell. Furthermore, the flux $Q$ across a small segment of a cell is also considered as shown in Fig. 2 (right) so that the zero-divergence condition is well satisfied in the fluid portion of the boundary cell.

A boundary cell contains the segment representing the body boundary in a variety of manner and the pressure computation is performed only in the cell of which pressure point is located in the fluid portion of the cell.

At each time step when the velocity field is updated the pressure field is regenerated in twofold stages. First the pressure in the region filled with $\mathrm{F}$-cells are computed by the SOR method being based on the Poisson equation. Secondly, keeping the pressure field of F-cells determined by the first stage as a Dirichlet condition, the pressure in the B-cells is determined by the simultaneous iterative method as described in details below. It is known that the simultaneous iterative method is basically equivalent to the SOR method ${ }^{4)}$ and that the former technique is very suitable for the pressure computation in boundary cells ${ }^{2)}$ since the pressure inside the boundary need not be postulated.

The simultaneous iterative method gives the following equation for the pressure.

$$
\phi_{i, k}^{m+1}=\phi_{i, k}^{m}-\frac{\omega}{2 \Delta t\left(\frac{1}{\Delta x^{2}}+\frac{1}{\Delta z_{k-1} \Delta z_{k}}\right)}\left[D_{i, k}\right]^{m}
$$

Here, $D$ is the divergence of the cell, $m$ is the iteration number and $\omega$ is the relaxation factor. The flux across a segment without a velocity point is estimated by approximately assuming the velocity profile to be expressed in the quadratic curves along the respective underlying grid lines. Then, this flux is considered in the calculation of $D$ in Eq. (13). The velocities on the side segments of a boundary cell are updated through the momentum equation by use of the pressure which is iteratively modified by Eq. (13).

\subsection{Other Boundary Conditions}

In the fluid flow problems containing the free-surface the exactly same technique with the TUMMAC-Vbk method $^{(0)}$ is employed. The free-surface geometry is approximated by a succession of segments in the similar manner with the segments of body boundaries. The segments are moved in a Lagrangian manner so that not only the overturning motion but also the impingement of the wave front on the other free-surface below and the vortical motion after breaking are approximately simulated $^{\text {7)10)11) }}$.

At the inflow boundary both velocity and pressure are set at the pre-determined values in the case of uniform stream. In the case of free-surface wave the inflow boundary works as a numerical wavemaker by giving oscillatory velocities and pressure derived from the linear wave theory.

At the outflow boundary the condition of zeronormal-gradient is employed, and similar open boundary conditions are imposed at the bottom boundary.

\section{Numerical test}

As a natural consequence of the use of a rectangular grid system the degree of the accuracy of the computation is deteriorated. However, the required degree of accuracy depends on the purpose of computation. In many fields of engineering the elucidation of a microscopic fluid phenomenon is not necessary but the understanding of fluid motions of larger scale is requested.

However, we must know the accuracy characteristics of the present method before applying to a variety of problems. Two test computations are performed for a flow past a circular cylinder under the condition listed in Table 1. Two cases at the Reynolds number $(R e) 10^{3}$ and $10^{5}$ are dealt with. The flow is accelerated for the period of 1.5 nondimensional time defined as

$$
T=t U / D \text {, }
$$

where $U$ is the uniform flow velocity and $D$ is the diameter of the cylinder.

The averaged pressure distribution at $R e=10^{3}$ is compared in Fig. 3. Even if a fine boundary-fitted coordinate system is employed ${ }^{14) 15)}$, noticeable discrepancy exists between the numerical simulation and the experiment. It is also noted from this figure that the

Table 1 Condition of computation for the numerical test with a circular cylinder.

\begin{tabular}{|l|c|}
\hline Diameter of circular cylinder, D (m) & 0.4 \\
\hline Reynolds number, Re & $10^{3}$ \\
\hline Domain of computation & $10^{5}$ \\
Length (m) & 3.000 \\
Height (m) & 1.554 \\
\hline Cell size & 10 \\
$\Delta x \quad(\mathrm{~mm})$ & $7 \sim 20$ \\
$\Delta z \quad(\mathrm{~mm})$ & 36,000 \\
\hline Approximate number of used cell & 2.00 \\
\hline Time increment, $\Delta t$ (millisecond) & 5.000 \\
\hline Total time steps & 600 \\
\hline Time steps for acceleration & 12.5 \\
\hline Nondimensional time, $T=t U / D$ & \\
\hline
\end{tabular}




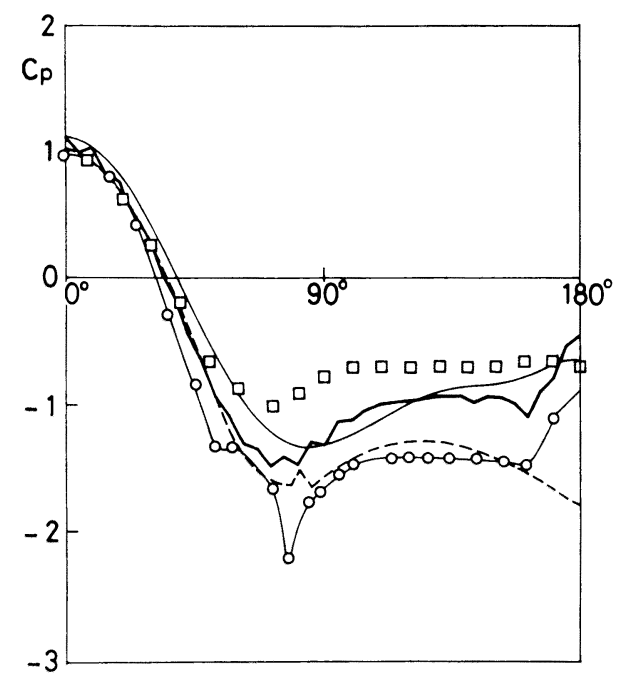

Fig. 3 Comparison of pressure distribution on a circular cylinder at $R e=1000,(-\mathrm{O}-)$; present method, $\square$; measured at $R e=2800$, from Goldstein $^{13)},(-,-)$; computed by Baba and Miyata at $R e=1000$ and 100 , respectively ${ }^{14)},(\cdots \cdots)$; computed by Kawamura and Kuwahara at $R e=$ $2000^{15)}$.

degree of agreement is raised by use of a fourth-order accurate compact scheme ${ }^{14)}$. It is shown that the present method is very slightly inferior to the method of third-order accuracy using a boundary-fitted coordinate system but that the abrupt change of pressure may be caused locally by the present method.

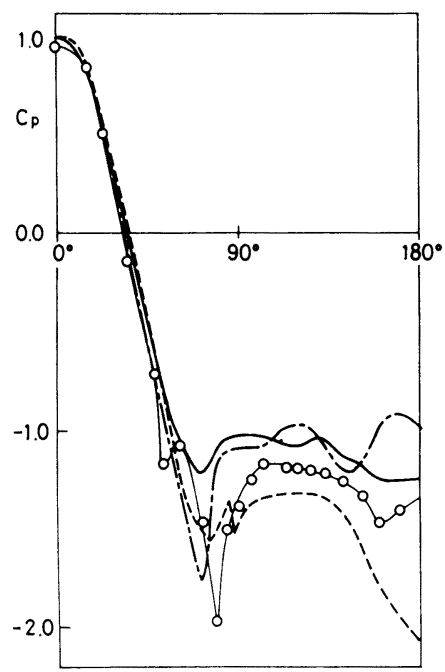

Fig. 4 Comparison of pressure distribution on a circular cylinder at $R e=10^{5}, \quad(-0-)$; present method, - ; measured by Cantwell and Coles at $\left.R e=1.4 \times 10^{5}{ }^{16}\right),(\cdots \cdots)$; measured by Nagamat. $\mathrm{su}$ at $R e=1.4 \times 10^{517)},(\cdots \cdot)$; computed by Kawamura and Kuwahara at $R e=10^{4}$ ).
The results for the case at $R e=10^{5}$ are shown in Fig. 4. Unrealistic variation of pressure is observed near the separation point but the overall degree of accuracy of the present method seems to be on the same level with the method using a boundary-fitted coordinate system.

\section{Some Applications}

Applicational computations are performed for three cases in which the geometry of the boundary is compli. cated. The first and second examples are a side-profile of an automobile and a pair of bridge section. One can generate a boundary-fitted coordinate system for these two cases, but one will find difficulties in removing the singular values at the sharp corners of these configurations. By the present method the solution is very conveniently obtained without any apprehension of solution break-down. The third case is a horizontal cylinder in waves. A moving grid system fitted both to the body boundary and to the free-surface can be generated such as one described for a 3D case in Ref. 18). However, the free-surface may cause wave breaking motion, to which a grid system fitted to the free-surface may find serious difficulties. The previous work ${ }^{5)} \mathrm{dem}$. onstrates that the method in a rectangular grid system can cope with the wave breaking motion. Therefore, the present method is useful for the simulation of a viscous flow past a body under the presence of the free-surface. The condition of computation is listed in Table 2 for the three cases.

\section{1 Flow about an Automobile}

The grid system in the vicinity of the automobile is shown in Fig. 5. The computation is started by giving a

Table 2 Condition of computation for some applica. tions.

\begin{tabular}{|c|c|c|c|}
\hline case & automobile & two bridges & $\begin{array}{c}\text { circular } \\
\text { cylinder with } \\
\text { free-surface }\end{array}$ \\
\hline $\begin{array}{l}\text { Length of body (diameter) } \\
\text { L. (m) }\end{array}$ & 4.0 & 18.0 & 0.16 \\
\hline Flow velocity, $U(\mathrm{~m} / \mathrm{s})$ & 10.0 & 20.0 & $\begin{array}{ll}\max & 0.102 \\
0.233 \\
0.328\end{array}$ \\
\hline Reynolds number, Re & $2.66 \times 10^{8}$ & $2.40 \times 10^{7}$ & $\begin{array}{l}16,890 \\
38,670 \\
54,380\end{array}$ \\
\hline $\begin{array}{l}\text { Domain of computation } \\
\text { Length (m) } \\
\text { Height (m) }\end{array}$ & $\begin{array}{r}20.000 \\
5.500\end{array}$ & $\begin{array}{l}43.950 \\
21.236\end{array}$ & $\begin{array}{l}1.992 \\
1.044\end{array}$ \\
\hline $\begin{array}{ll}\text { Cell size } & \\
\Delta x & (\mathrm{~mm}) \\
\Delta z & (\mathrm{~mm})\end{array}$ & $\begin{array}{c}80 \\
20 \sim 100\end{array}$ & $\begin{array}{c}150 \\
50 \sim 250\end{array}$ & $\begin{array}{c}6 \\
3 \sim 8\end{array}$ \\
\hline $\begin{array}{l}\text { Approximate number of } \\
\text { used cell }\end{array}$ & 25,000 & 45,000 & 72,000 \\
\hline $\begin{array}{l}\text { Time increment } \\
\text { (millisecond) }\end{array}$ & 0.40 & 0.36 & $\begin{array}{l}1.25 \\
0.55 \\
0.39\end{array}$ \\
\hline Total time steps & 5,000 & 5,000 & $\begin{array}{r}7,840 \\
17,940 \\
20,372\end{array}$ \\
\hline $\begin{array}{l}\text { Time steps for } \\
\text { acceleration }\end{array}$ & 1,000 & 1,700 & .. \\
\hline $\begin{array}{l}\text { Nondimensional time } \\
T=t U / L\end{array}$ & 5.0 & 2.0 & $\begin{array}{r}6.25 \\
14.37 \\
16.20\end{array}$ \\
\hline
\end{tabular}




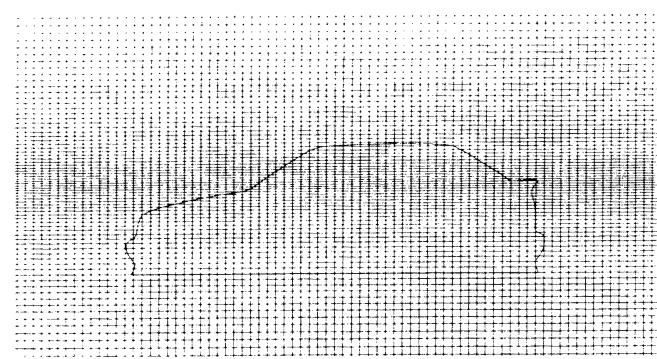

Fig. 5 Grid system in the vicinity of an automobile.

number of coordinates that represent the automobile profile and the result is obtained within a day. The computed results are shown in Figs. 6 and 7. It is noted that the pressure is made dimensionless with respect to
$1 / 2 \rho U^{2}$ for the cases without free-surface. Since the ground and wheels are eliminated and the flow is assumed to be two-dimensional, the simulated flow is not of real automobile. However, it may be safe to say that the present method is useful for the understanding of the overall flow field about a body of complex geometry. One of the advantages of the present method is that the degree of accuracy is not deteriorated in the region far behind the automobile in contrast with the result in a body-fitted coordinate system ${ }^{23)}$ which provides very coarse resolution of the flow far away from a body.

\section{2 Flow about Two Bridges}

The grid system about two bridge sections is shown in Fig. 8. The uniform wind speed is assumed to be $20 \mathrm{~m} /$ $\mathrm{s}$ and consequently the Reynolds number based on the length in Table 2 is as high as $2.4 \times 10^{7}$. The computed
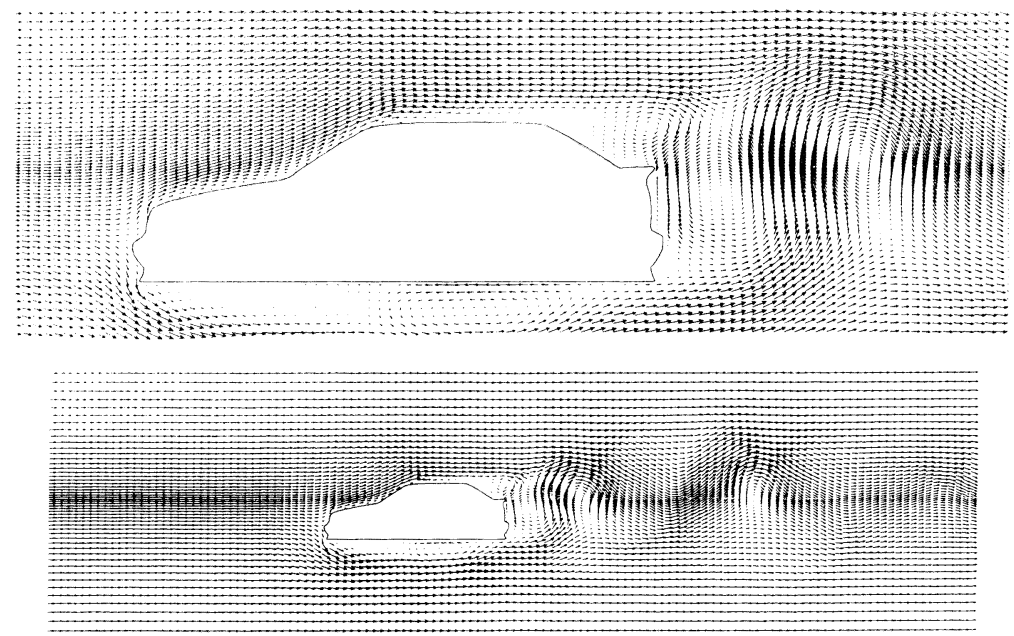

Fig. 6 Velocity vector fields about an automobile at $T=5$.
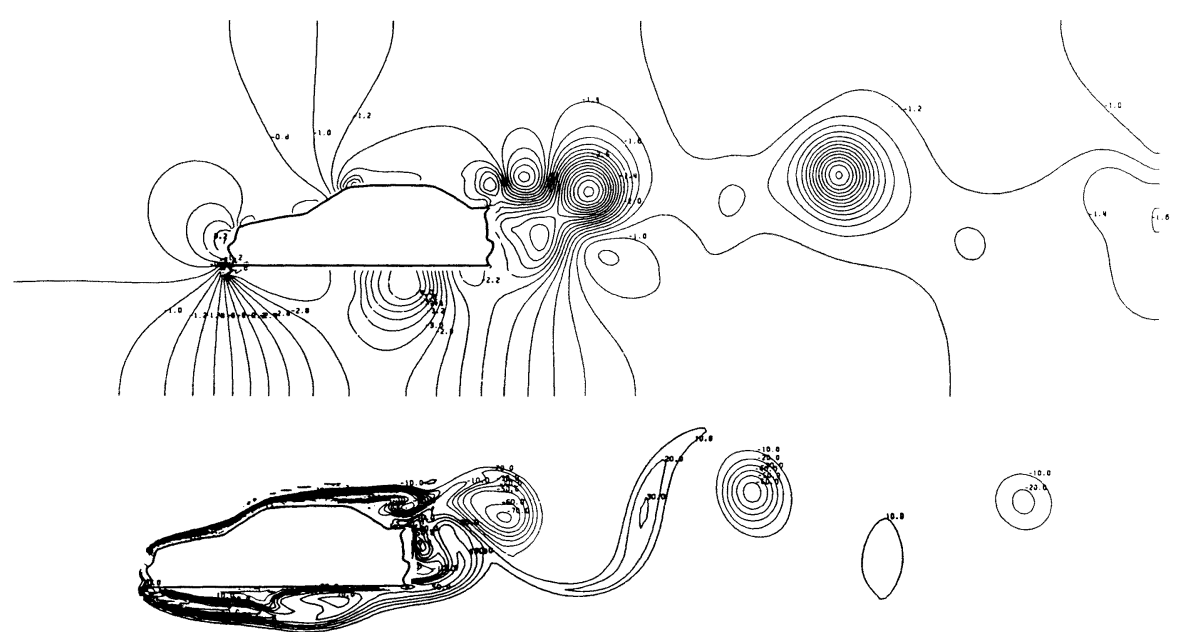

Fig. 7 Pressure and vorticity contour maps about an automobile at $T=5$, positive pressure coefficient and vorticity of anticlockwise rotation are drawn in bold lines, the contour interval is 0.2 for pressure and $10 / \mathrm{s}$ for vorticity. 


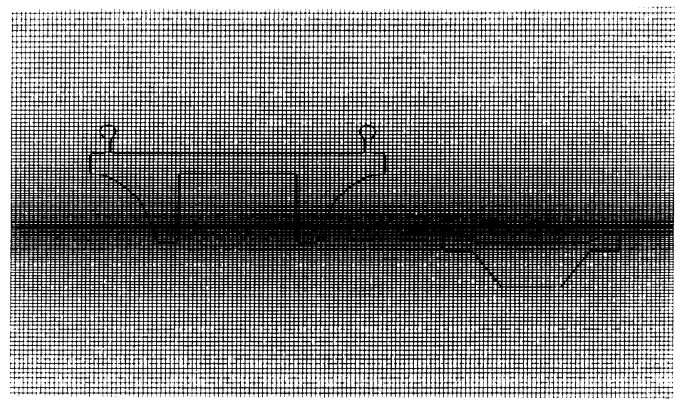

Fig. 8 Grid system in the vicinity of two bridge sections.

results are shown in Figs. 9 to 11 . Since the nondimensional time is only 2 at the final stage, the shed vortices are not fully developed. However, it is obviously noted that the complicated vortex motions are well simulated by the present method. A variety of vortex interactions are present. It is imagined that it may be very difficult to have this result by flow visualization.

\section{3 Flow about a Circular Cylinder in Free-} Surface Waves

Forces on a circular cylinder placed horizontally beneath the free-surface with its axis parallel to the crests are caused by both wave motion and the viscous motion. When the $K_{c}$ number $(=U m T / D$, where $U m$ maximum velocity, $T$ wave period and $D$ diameter) is much smaller than unity, the viscous motion can be ignored because the forces are mostly caused by the

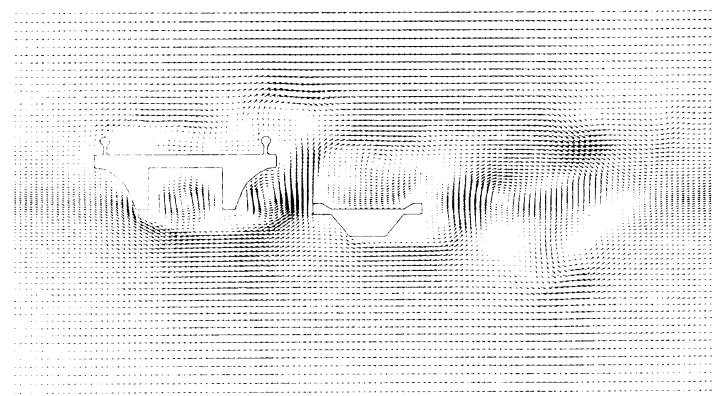

Fig. 9 Velocity vector field at $T=2$.

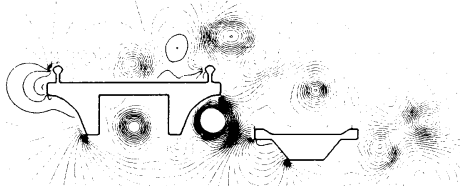

Fig. 10 Pressure contour map about two bridge sections at $T=2$, positive values of pressure coefficient are drawn in bold lines, the contour interval is 0.2 .
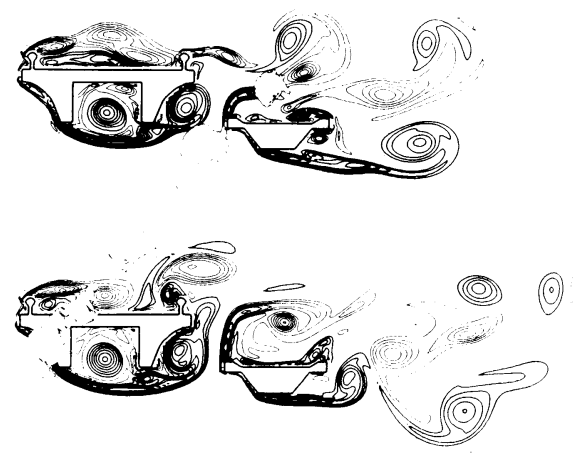

Fig. 11 Vorticity contour map about two bridge sections at $T=1.6$ (above) and 2.0 (below), anticlockwise vorticity is contoured in bold lines and the contour interval is $10 / \mathrm{s}$.

pressure field due to the waves. However, when $K_{c}$ exceeds unity, the viscous motion plays an important role in this probiem. The drop of inertia forces at $K_{c}=$ 1 to 4 is explained as a result of generating circulation about the cylinder ${ }^{20) 21)}$. However, the mechanism is still left vague, and furthermore, one does not have any useful tool of force estimation except experiment. This is one of the problems to which the present method may be proved very useful, because it simulates wave and viscous motions simultaneously.

The grid system is shown in Fig. 12. Fine spacing in the vertical direction is distributed near the free-surface

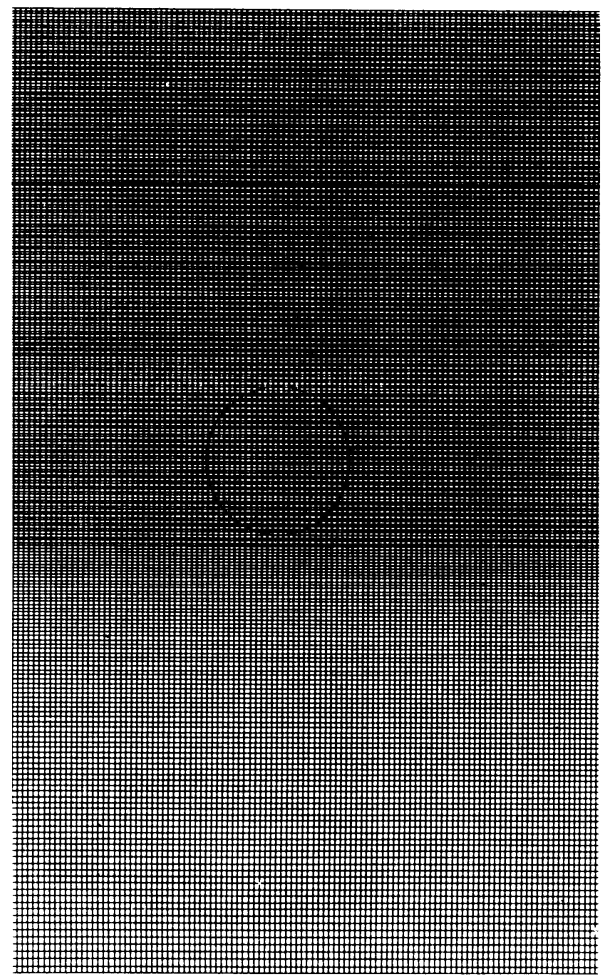

Fig. 12 Grid system in the vicinity of a circular cylinder and a free-surface. 
as well as the circular cylinder. Three cases at $K_{C}=$ $1.20,2.75$ and 3.86 are chosen for the computation. The wave length is kept constant at $5.5 \mathrm{~m}$ and the wave height, $0.086 \mathrm{~m}, 0.197 \mathrm{~m}$ and $0.277 \mathrm{~m}$, corresponds to the three cases of $K_{c}$ number through the maximum flow velocity at the center of the cylinder which is located $0.30 \mathrm{~m}$ below the undisturbed free-surface. The condition of computation is listed in Table 2.

The simulated flow field for the case of $K_{C}=2.75$ is shown in Fig. 13, in which the vortex generation is significant. It is obvious that the pressure field caused by the sinusoidal wave motion is remarkably distorted by the vortex motions, which implies that the forces, given by integrating the pressure distribution on the cylinder, are significantly influenced by the viscous flow. Since the $K_{c}$ number is not very large, the vortices generated on the surface of the cylinder are not shed far from the cylinder but they stay in the vicinity of the cylinder and interact each other in an extremely complicated manner.

The inertia force coefficients defined below are compared with experimental results in Fig. 14, in which the computed results are plotted for the two cases for which computation is continued for a sufficient number of wave oscillations. The agreement is good, which implies that the present method can resolve most of the complicated fluid motion of this problem.

$$
\begin{aligned}
& C_{m x}=f_{m x} /\left\{h \sigma^{2} \rho A L \exp (-W d)\right\}, \\
& C_{m z}=f_{m z} /\left\{h \sigma^{2} \rho A L \exp (-W d)\right\} .
\end{aligned}
$$

Here, $C_{m x}$ and $C_{m z}$ are the intertia force coefficients, $f_{m x}$ and $f_{m z}$ are respective forces, $h$ is the wave amplitude, $\sigma$ is the wave frequency, $A$ is the sectional area, $L$ is the spanwise length of a cylinder, $W$ is the wave number and $d$ is the depth of submergence.

\section{Concluding Remarks}

It is demonstrated in this paper that a finite-difference simulation method in a rectangular grid system has some advantages for the simulation of a flow with boundaries of complex geometry and that the approximate no-slip body boundary conditions work well without unfavorable behaviors. The present method will be

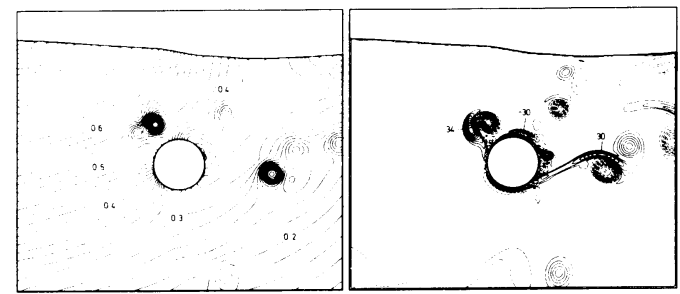

Fig. 13 Pressure (left) and vorticity (right) contour maps at $K_{C}=2.75$, the hydrostatic pressure is subtracted and positive pressure is drawn in solid lines, the contour interval is $0.02 \mathrm{~m}^{2} / \mathrm{s}^{2}$. Anticlockwise vorticity is contoured in solid lines and the contour interval is $4 / \mathrm{s}$.
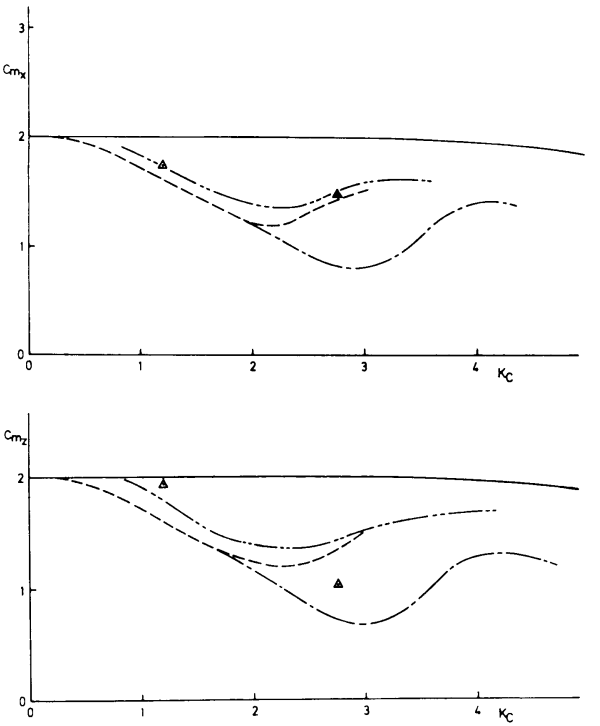

Fig. 14 Horizontal and vertical force coefficients, $\Delta$; present method, (-) ; measured by Sarpkaya and Isaacson in plane oscillatory flow ${ }^{19}$, $(\cdots .$.$) ; measured by$ Chaplin $^{20)},(---)$; measured by Ikeda et al. $^{21)},(-\cdots-)$; measured by Miyata et al. ${ }^{22)}$.

useful to a variety of flow simulations with practical purposes.

The computations were executed by a mainframe computer HITAC M-680 H of the Computer Center, the University of Tokyo. The CPU time was from 4 to 25 hours for the computations described in Section 6. The present work is partly supported by the Grant-in-Aid for Cooperative Research of the Ministry of Education, Science and Culture and also by the LINEC research group of shipbuilders in Japan. The authors are thankful to Professor H. Kajitani for his encouragement and also to Ms. M. Fujita and K. Onoda for their careful typewriting.

\section{References}

1) J. E. Welch, F. H. Harlow, J. P. Shannon, and B. J. Daly, "The MAC Method", Los Alamos Scientific Lab. Report LA-3425, Los Alamos, N. M., 1966.

2) J. A. Viecelli, J. Comput. Phys. 8 (1971), 119.

3) H. Miyata, and S. Nishimura, J. Fluid Mech. 157 (1985), 327-357.

4) H. Miyata, S. Nishimura, and A. Masuko, J. Comput. Phys. 60 (1985), 391-436.

5) H. Miyata, J. Comput. Phys. 65 (1986), 179-214.

6) H. Miyata, M. Katsumata, Y.-G. Lee, and H. Kajitani, J. Soc. Naval Arch. Japan 163 (1988), 1 -16 .

7) H. Miyata, S. Nishimura, and H. Kajitani, "Proc. 4th International Conference on Numerical Ship Hydrodynamics", pp. 259 - 290 (1985), David Taylor Naval Ship R\&D Center.

8) H. Miyata, C. Matsukawa, and H. Kajitani, J. 
Soc. Naval Arch. Japan 158 (1985), 11-23.

9) H. Miyata, H. Kajitani, M. Zhu, T. Kawano, and M. Takai, J. Kansai Soc. Naval Arch., Japan, 207 (1987), 11-23.

10) H. Miyata, Comput. Mech. 3 (1988), 217-228.

11) H. Miyata, H. Kajitani, M. Zhu, and T. Kawano, "Proc. 16th Symposium on Naval Hydrodynamics", pp. 514-534 (1986), National Academy Press.

12) J. W. Deardorff, J. Fluid Mech. 41 (1970), pp. 453480.

13) S. Goldstein, "Modern Developments in Fluid Dynamics", Oxford, 1938.

14) N. Baba and H. Miyata, J. Comput. Phys. 69 (1987), pp. 362-396.

15) T. Kawamura and K. Kuwahara, AIAA paper 84 -0340, 1984 (unpublished).

16) B. Cantwell and D.Coles, J. Fluid Mech. 136
(1983), pp. 321-374.

17) T. Nagamatsu, "Proc. 1st. Symposium of Ship Propulsion Committee", Soc. Naval Arch. Japan, 1985.

18) H. Miyata, T. Sato and N. Baba, J. Comput. Phys. 72 (1987), 393-421.

19) T. Sarpkaya and M. Isaacson, "Mechanics of Wave Forces on Offshore Structures", Van Nostrand Reinhold Company, 1981.

20) J. R. Chaplin, J. Fluid Mech. 140 (1984), pp. 175187.

21) Y. Ikeda, K. Otsuka and N. Tanaka, J. Soc. Naval Arch. Japan, 163 (1988), pp. 214-221.

22) H. Miyata, N. Shikazono and M. Kanai, Ocean Engineering (to appear) (1989).

23) Proc. Third Symposium on Numerical Fluid Dynamics, Chuo Univ. (1989). 